\title{
Optimization of Antennas using a Hybrid Genetic-Algorithm Space-Mapping Algorithm
}

\author{
Pantoja, M.F.; Bretones, A.R.; Meincke, Peter; Garcia, S.G.; Martin, R.G.
}

Published in:

Proceedings of the EuCAP - European Conference on Antennas and Propagation

Publication date:

2006

Document Version

Publisher's PDF, also known as Version of record

Link back to DTU Orbit

Citation (APA):

Pantoja, M. F., Bretones, A. R., Meincke, P., Garcia, S. G., \& Martin, R. G. (2006). Optimization of Antennas using a Hybrid Genetic-Algorithm Space-Mapping Algorithm. In Proceedings of the EuCAP - European Conference on Antennas and Propagation (pp. 346982)

http://server.oersted.dtu.dk/publications/views/publication_details.php?id=2893

\section{General rights}

Copyright and moral rights for the publications made accessible in the public portal are retained by the authors and/or other copyright owners and it is a condition of accessing publications that users recognise and abide by the legal requirements associated with these rights.

- Users may download and print one copy of any publication from the public portal for the purpose of private study or research.

- You may not further distribute the material or use it for any profit-making activity or commercial gain

- You may freely distribute the URL identifying the publication in the public portal 


\title{
OPTIMIZATION OF ANTENNAS USING A HYBRID GENETIC-ALGORITHM SPACE-MAPPING ALGORITHM
}

\author{
M. F. Pantoja* , A. R. Bretones*, Peter Meincke ${ }^{\circ}$, S. G. García*, and R. G. Martín* \\ *Facultad de Ciencias, Departamento de Electromagnetismo, University of Granada, 18071 Granada, Spain \\ ${ }^{\circ}$ Technical University of Denmark, Ørsted-DTU, Electromagnetic Systems, DK-2800 Lyngby, Denmark
}

\begin{abstract}
A hybrid global-local optimization technique for the design of antennas is presented. It consists of the subsequent application of a Genetic Algorithm (GA) that employs coarse models in the simulations and a space mapping (SM) that refines the solution found in the previous stage. The technique is particularly suited to optimization problems for which long computational times are required to achieve accurate solutions.
\end{abstract}

Key words: Optimization methods; genetic algorithms; space mapping; antenna arrays.

\section{INTRODUCTION}

The application of genetic algorithms (GAs) as optimization tools for the design of antennas has been an active field of research in the past decade (Rahmat-Samii \& Michielssen 1999). The main reasons for this interest are related to their robustness, enabling the solution of optimization problems for which local techniques of optimization are not effective, as well as their versatility, permitting the successful use of the same schemes to different problems (Haupt 1995).

There are, however, inherent restrictions to the applicability of the GAs. As a consequence of their structure, the problems, for which high computational times are needed to accurately simulate each possible solution, remain yet excessively costly. To overcome this problem, several efforts have been devoted to find more efficient optimization schemes, resulting not only in improved versions of the genetic algorithms, e.g. micro-genetic algorithms $(\mu \mathrm{GA})$ (Krishnakumar 1989) and hybrid taguchi genetic algorithms (Tsai et al. 2004), but also in new global techniques of optimization derived from different philosophies, e.g. particle swarm optimizations (Kennedy \& Eberhart 2001) and ant colony optimizations (Coleman et al. 2004). These improvements, along with the increas- ing capability of computers and the development of parallel codes (Levine 1995), have led to satisfactory solutions for more complex problems. Moreover, in problems for which the accuracy of the optimized solution is not critical, a usual procedure for decreasing the total computational time is to reduce the computational burden of the models by, for example, using a coarse meshing of the computational grid in simulators based on finite element methods (Mohammed 1999) or decreasing the number of basis functions in codes applying the method of moments (Fernández-Pantoja et al. 2000). Unfortunately, an estimation of the error introduced by these approaches is often difficult to make.

In this communication, an additional stage is introduced in the optimization procedure to assure the accuracy of the final result. This additional step, based on the spacemapping (SM) technique (Bandler et al. 2004), allows GA operators to employ coarse models in the simulations to find an approximate solution of the problem. Once attained, SM provides an accurate solution of the problem with a relatively low computational cost. SM techniques, in conjunction with different local optimization problems, have previously proven effective for solving different optimization problems in electromagnetics (Bakr 2000), (Bandler et al. 1995).

As an example of optimization the hybrid algorithm GA$\mathrm{SM}$ is used to select the lengths and feeding points of an array of $3 \times 3$ patch antennas on a finite ground plane. Results and graphs of this particular case will be shown on the poster presentation of this communication.

\section{HYBRID GA-SM OPTIMIZATION ALGO- RITHM}

A flowchart of the algorithm, presented in Figure 1, consists basically of two different strategies applied consecutively. First, a GA optimizer offers, by means of repetitive, fast computer simulations of possible solutions, a low-accuracy optimal resolution of the problem. This in- 
termediate result is referred to as a coarse optimal solution. Second, an accurate simulation of the coarse optimal solution is performed to verify whether the coarse solution is acceptable to provide a final solution to the problem. If this accurate analysis shows unsatisfactory characteristics, i.e., displacements of the resonance frequencies or increased levels of input reflection coefficients, a subsequent stage based on SM is initiated. In this stage, a local optimizer using both coarse and fine models is employed to produce an accurate solution of the problem, denoted by the fine optimal solution. This solution resembles the coarse solution offered by GA in those parameters chosen to be the objectives of the optimization. Consequently, the designer must define the parameters that vary in the optimization process, denoted by $\bar{x}_{c}$ and $\bar{x}_{f}$, and the characteristics (basis functions, precision of integrals, etc.) of both coarse and fine models. The correct choices at this point will be essential for the success of the optimization. The coarse model should be as fast as possible, but keeping a certain similarity between its response $R_{c}\left(\bar{x}_{c}\right)$ and the response $R_{f}\left(\bar{x}_{f}\right)$ offered by the fine model. Otherwise, the SM stage will not work properly. Once selected, the GA optimizer provides the optimal coarse solution, denoted by $\bar{x}_{c}^{*}$, by means of applying genetic operators only over coarse simulations. Once determined that the deviation of the response calculated by an accurate simulation $R_{f}\left(\bar{x}_{c}^{*}\right)$ of the optimal coarse solution is higher than accepted, the SM algorithm seeks for a mapping $\tilde{P}$ between the fine and coarse models $\bar{x}_{c}=\tilde{P}\left(\bar{x}_{f}\right)$, so that $R_{f}\left(\bar{x}_{f}\right) \approx R_{c}\left(\bar{x}_{c}\right)$. To determine $\tilde{P}$, an iterative local optimization is performed. The key steps in the SM algorithm are the parameter-extraction phase, which ascertains the coarse model that better fits a certain fine model, the update-mapping level, which alters the estimate of $\tilde{P}$ using a Broyden equation (Bandler et al. 1995), and the invert-mapping level, which determines the fine model for the next iteration. If the coarse and fine models are properly chosen, this iterative process converges at the fine optimal solution $\bar{x}_{f}^{*}$ when the responses $R_{f}\left(\bar{x}_{f}\right)$ and $R_{c}\left(\bar{x}_{c}\right)$ are similar up to a previously fixed level of precision. More details on the SM can be found in (Bandler et al. 2004).

\section{EXAMPLE OF OPTIMIZATION}

To test the adequacy of the method, the determination of proper lengths and feeding points for an array of $3 \times 3$ patch antennas on a finite square ground plane to operate at a frequency of $4.5 \mathrm{GHz}$ is proposed as an example of optimization. Given the symmetry of the problem, as indicated in Figure 2(a), there are a total of 12 optimization parameters, related both to the lengths $\left(L_{1}, \ldots, L_{6}\right)$ and to the distances of the feeding points from the center of the patch $\left(d_{1}, \ldots, d_{6}\right)$. Fixed quantities of this exam-

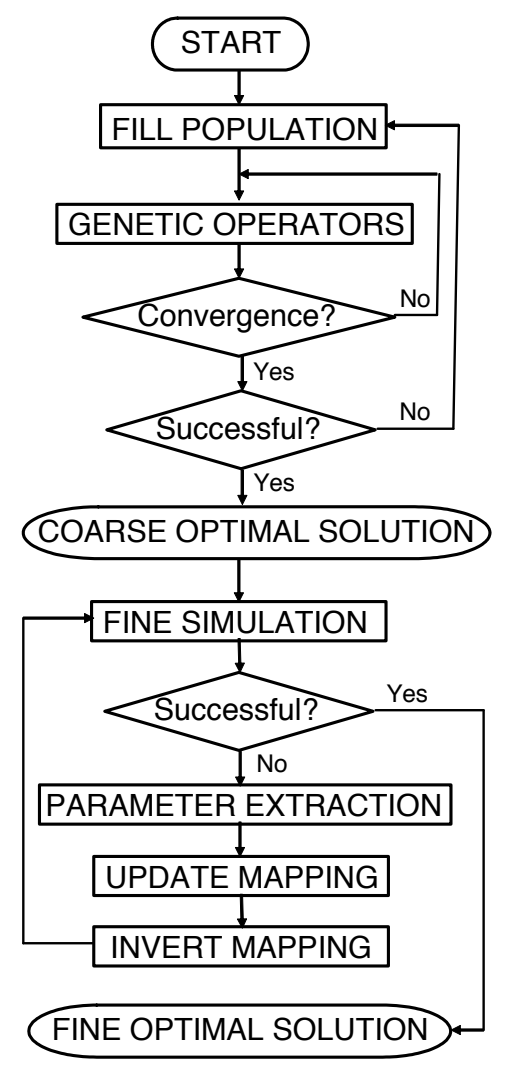

Figure 1. Flowchart of the hybrid GA-SM algorithm.

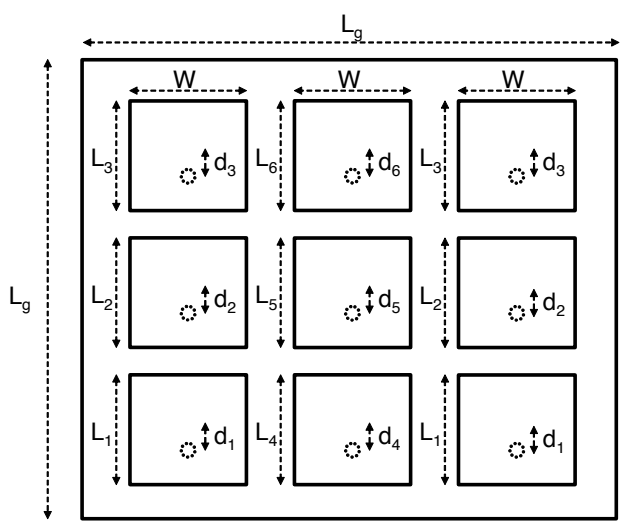

(a) Top view

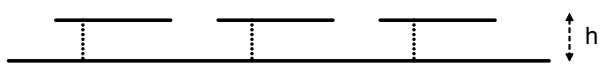

(b) Side view

Figure 2. Array of patch antennas on finite ground plane. 
ple are the widths of the patches $(W=3 \mathrm{~cm})$, the side length of the ground plane $\left(L_{g}=12 \mathrm{~cm}\right)$, and the distance between the antennas and ground $(h=0.15 \mathrm{~cm})$. The substrate used is air.

The use of a global optimizer to solve this problem requires a reliable code to simulate randomly generated designs. All the results shown in this paper are derived from the method-of-moments solution of the mixed-potential electric-field integral equation with higher-order Legendre basis functions (Jørgensen et al. 2004). Given the specific set of lengths and feeding points described above, an accurate solution of this problem requires 6000 basis functions with an analysis time of 4 minutes per frequency on a $2.2 \mathrm{GHz}$ AMD Opteron processor. As the search space consists of $10^{12}$ possible solutions to be considered in the optimization process, the optimization results are reached with a $\mu \mathrm{GA}$ algorithm after approximately 3000 simulations. If no parallelization is applied, the total optimization time for this simple case could be about nine days. The application of a coarse model of the individuals, consisting of a decrease in both the number of basis functions and precision of the integrals which is described in the following section, leads to a faster result with the cost of a displacement in the frequency spectrum of the simulated response of some $100 \mathrm{MHz}$.

Thus, the GA-SM optimization was performed. The GA stage, using only coarse models of the array, was conducted by means of an elitist $\mu G A$ algorithm (Krishnakumar 1989), with a population of 5 individuals and a $80 \%$ convergence for a replacement of the population. The GA operators employed were a tournament selection and a double-point crossover (Back et al. 1997). Possible solutions were generated using fixed-point codification, with a total of 12 integer digits ranging from $3 \mathrm{~cm}$ to $3.25 \mathrm{~cm}$ for the lengths and from $0.33 \mathrm{~cm}$ to $0.60 \mathrm{~cm}$ for the distance of the feeding points to the center of the patch. The values allowed for the patch lengths in the GA process were established using approximate equations for the resonance frequency of rectangular microstrip patch antennas over infinite ground plane, ranging from $4.4 \mathrm{GHz}$ to $4.8 \mathrm{GHz}$, these corresponding, respectively, to $3.25 \mathrm{~cm}$ and $3 \mathrm{~cm}$. The fitness function $F$ was designed to minimize at $4.5 \mathrm{GHz}$ the maximum value of the magnitude of the input reflection coefficient for any antenna of the array $\left(F=\max \left\{\left|S_{11}\right|_{i}\right\} ; i=1, \ldots, 6\right)$. Figure 3 shows the magnitude of the input reflection coefficient of each antenna element in this coarse solution, indicating different resonance frequencies for each patch antenna, but all of them near the desired $4.5 \mathrm{GHz}$ of operation.

Nevertheless, a simulation with a fine model of the same antenna reveals a shift in the spectrum of approximately $130 \mathrm{MHz}$ (see Figure 4). For a correction of this effect, the SM stage is introduced. The fine space was defined with only two parameters $\bar{x}_{f}$, each one to scale

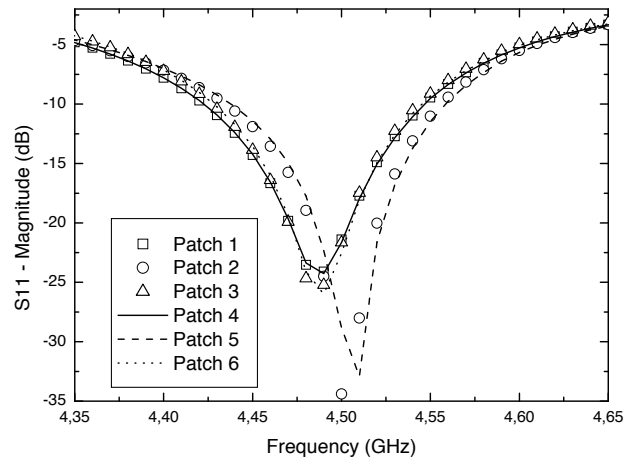

Figure 3. Input reflection coefficients based on the coarse optimal solution.

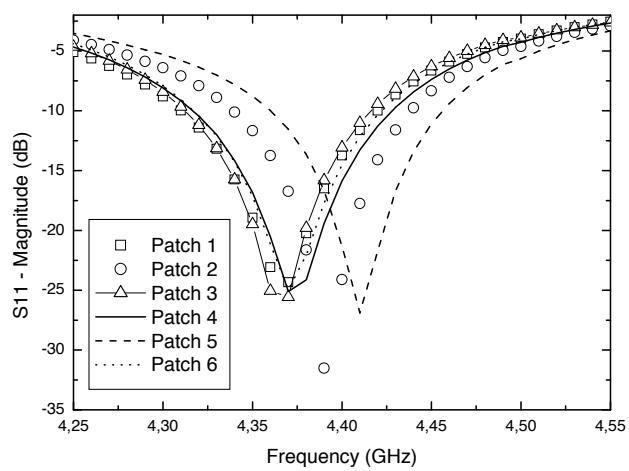

Figure 4. Input reflection coefficients based on an accurate simulation of the coarse optimal solution.

respectively the lengths and the distances from the feeding points, respectively, of the optimal coarse solution. As pointed out in (Bakr 2000), the convergence of the model is better achieved when several frequency points of analysis are employed. In this case, 11 frequency points were distributed between $4.25 \mathrm{GHz}$ to $4.75 \mathrm{GHz}$. The parameter-extraction phase was accomplished following the aggressive space-mapping approach (Bandler et al. 1995), and taking, as a measure of the similarity between the fine and coarse models, the mean square error of the difference between their respective real parts of the input impedance, for all the patches along the frequency points of the analysis. Other relevant selections in this SM stage were the stopping criteria, set to $10^{-4}$, and the numerical estimate of the analytical Jacobian by using a forward-difference approximation. A key point to achieve a quick convergence is to evaluate the similarity between fine and coarse-model responses using as measuring function the real part of the input impedance rather than the magnitude of input reflection coefficient, due to the fact that the greater smoothness of the former leads to better forward-difference approximations. After a total of three fine simulations and 45 coarse simulations, the algorithm converged to the final solution. Figure 5 shows the effective correction of the operational point to the 4.5 $\mathrm{GHz}$. 


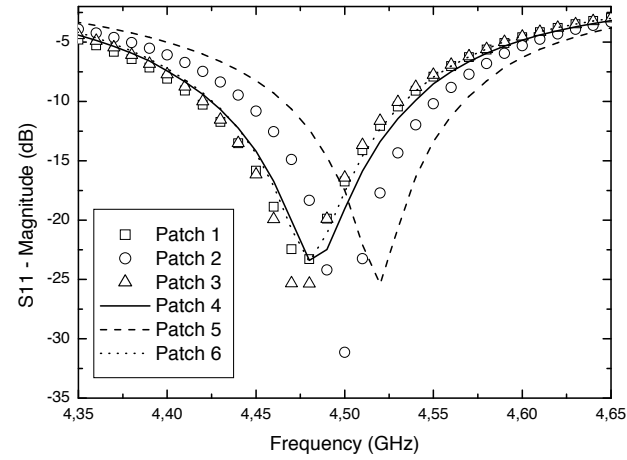

Figure 5. Input reflection coefficients based on the fine optimal solution.

Finally, an estimation is made of the time saved by using the GA-SM method, in contrast to the GA method using only fine simulations. Given that the time consumption for the fine model in this case is 6.5 times slower per frequency than the coarse model, and that each fine or coarse simulation made in the SM stage solved 11 frequency points, the optimization was performed roughly 5.25 times faster applying this hybrid technique. Hence, as long as the limit on the time reduction depends on the difference between the analysis time of fine and coarse models, the designer has to look for faster coarse models to achieve greater reductions. In any event, this process should be carried out carefully since the SM stage works only effectively if the response of the coarse model is similar to that of the fine model.

\section{CONCLUSION}

In this communication, an efficient scheme has been proposed for the optimization of antennas. This scheme consists of applying an additional space-mapping technique after a genetic-algorithm optimization, the latter employing a coarse model in the simulation of the antenna response. The SM stage of the process improves the accuracy of the optimized results, and the total approach has been demonstrated to offer advantages in terms of computational cost over the single application of GA with a fine-model simulator. Further studies will be conducted to compare the performance of the GA-SM method with other hybrid methods combining local optimization techniques and SM.

\section{ACKNOWLEDGMENTS}

This work was supported by the "Secretaría de Estado de Universidades e Investigación del Ministerio de Educación y Ciencia" through "Programa Nacional de ayudas para la movilidad de profesores de universidad" as well as the Danish Technical Research Council and Spanish Ministerio de Ciencia y Tecnologa, under Projects TEC2004-06217-C02-01 and TEC-2004-04866-C04-03.

\section{REFERENCES}

Back, T., Fogel, D., \& Michalewicz, Z., eds. 1997, Handbook of evolutionary computation (Bristol, UK: IOP Publishing)

Bakr, M. H. 2000, PhD thesis, Univerity of Hamilton

Bandler, J. W., Biernacki, R. M., Chen, S. H., Hemmers, R. H., \& Madsen, K. 1995, IEEE Trans. Microwave Theory Tech., 43, 2874

Bandler, J. W., Cheng, Q. S., Dakroury, S. A., et al. 2004, IEEE Trans. Microwave Theory Tech., 52, 337

Coleman, C., Rothwell, E., \& Ross, J. 2004, IEEE Trans. Antennas Propagat., 52, 1007

Fernández-Pantoja, M., Monorchio, A., Rubio-Bretones, A., \& Gómez-Martín, R. 2000, Electronics Letters, 36, 1988

Haupt, R. L. 1995, IEEE Antennas Propagat. Mag., 37, 7

Jørgensen, E., Volakis, J. L., Meincke, P., \& Breinbjerg, O. 2004, IEEE Trans. Antennas Propagat., 52, 2985

Kennedy, J. \& Eberhart, R. C. 2001, Swarm Intelligence (San Francisco: Morgan Kauffman)

Krishnakumar, K. 1989, in SPIE: Intelligent Control and Adaptive Systems

Levine, D. 1995, Users Guide to the PGAPACK Parallel Genetic Algorithm Library, Tech. rep. anl-95/18, Argonne National Laboratory

Mohammed, O. A. 1999, in Electromagnetic Optimization by Genetic Algorithms, ed. Y. Rahmat-Samii \& E. Michielssen (John Wiley \& Sons)

Rahmat-Samii, Y. \& Michielssen, E., eds. 1999, Electromagnetic Optimization by Genetic Algorithms (New York, N.Y.: John Wiley \& Sons)

Tsai, J. T., Liu, T. K., \& Chou, J. H. 2004, IEEE Trans. Evol. Comput., 8, 365 